livraisons

d'Histoire

de l'Architecture

\section{Livraisons de l'histoire de l'architecture}

$35 \mid 2018$

Femmes, architecture et paysage

\title{
L'ordre des femmes à la renaissance
}

The order of the women in the Renaissance

Weibliche Säulenordnungen in der Renaissancezeit

\section{Yves Pauwels}

\section{OpenEdition}

\section{Journals}

Édition électronique

URL : http://journals.openedition.org/lha/904

DOI : 10.4000/lha.904

ISSN : 1960-5994

Éditeur

Association Livraisons d'histoire de l'architecture - LHA

Édition imprimée

Date de publication : 15 juin 2018

Pagination : 17-28

ISSN : $1627-4970$

\section{Référence électronique}

Yves Pauwels, "L'ordre des femmes à la renaissance », Livraisons de l'histoire de l'architecture [En ligne], 35 | 2018, mis en ligne le 15 juin 2020, consulté le 24 janvier 2021. URL : http:// journals.openedition.org//ha/904; DOl : https://doi.org/10.4000//ha.904 
Par Yves Pauwels

\section{L'ORDRE DES FEMMES À LA RENAISSANCE}

En 1567, dans son Premier tome de l'architecture, Philibert De l'Orme écrit à propos du château des Tuileries qu'il est en train de construire pour Catherine de Médicis (ill. 1) :

L'autre raison pourquoi j'ai voulu figurer et naturellement représenter ledit ordre ionique au palais de la majesté de la reine, c'est pour autant qu'il est féminin, et a été inventé après les proportions et ornements des dames et déesses, ainsi que le dorique des hommes, comme m'ont appris les anciens : car quand ils voulaient faire un temple à quelque dieu, ils y employaient l'ordre dorique : et à une déesse, le ionique. [...] Je me suis donc justement voulu aider au susdit palais de la majesté de la reine de l'ordre ionique, comme étant délicat, et de plus grande beauté que le dorique, et plus orné et enrichi de singularités. Car l'ordre dorique de soi, pour être masculin est plus rude, et semble avoir été inventé pour choses fortes, à fin de soutenir grands poids et grands fardeaux (ainsi que nous avons dit auparavant) et porter grandes hauteurs de maçonnerie, comme aux châteaux et forteresses, sans guère d'ornements. ${ }^{1}$

En liant ainsi les ordres dorique et ionique aux qualités supposées des deux sexes, De l'Orme ne se laisse pas aller à une divagation qui pourrait aujourd'hui paraître quelque peu saugrenue de la part d'un architecte parfaitement raisonnable par ailleurs. Au contraire, et ceci nous oblige à le prendre au sérieux, il s'inscrit dans une antique tradition qui remonte à Vitruve ${ }^{2}$. Ce dernier, lorsqu'il évoque la généalogie des ordres au livre IV du De architectura, explique que les Ioniens, pour édifier un temple à Apollon, reprenant pour en l'améliorant la forme inventée "par hasard " (fortuito) par les Doriens,

dimensi sunt virilis pedis vestigium et id retulerunt in altitudinem. Cum invenissent pedem sextam partem esse altitudinis in homine, idem in columnam transtulerunt et qua crassitudine fecerunt basim scapi, tantas sex

1. Premier tome de l'architecture, f. $155 \mathrm{v}^{\mathrm{o}}-156$. Je modernise la graphie, comme pour toutes les autres citations des $\mathrm{XVI}^{\mathrm{e}}$ et $\mathrm{XVII}{ }^{\mathrm{e}}$ siècles. L'ouvrage, ainsi que tous ceux mentionnés dans cet article, est consultable en ligne sur le site ARCHITECTURA du Centre d'études supérieures de la Renaissance à Tours (http://architectura.cesr.univ-tours.fr/).

2. Sur la généalogie des ordres chez Vitruve et ses développements à l'époque moderne, voir Y. Pauwels, Aux marges de la règle. Essai sur les ordres d'architecture à la Renaissance. Wavre, Mardaga, 2008, p. 28-38. 


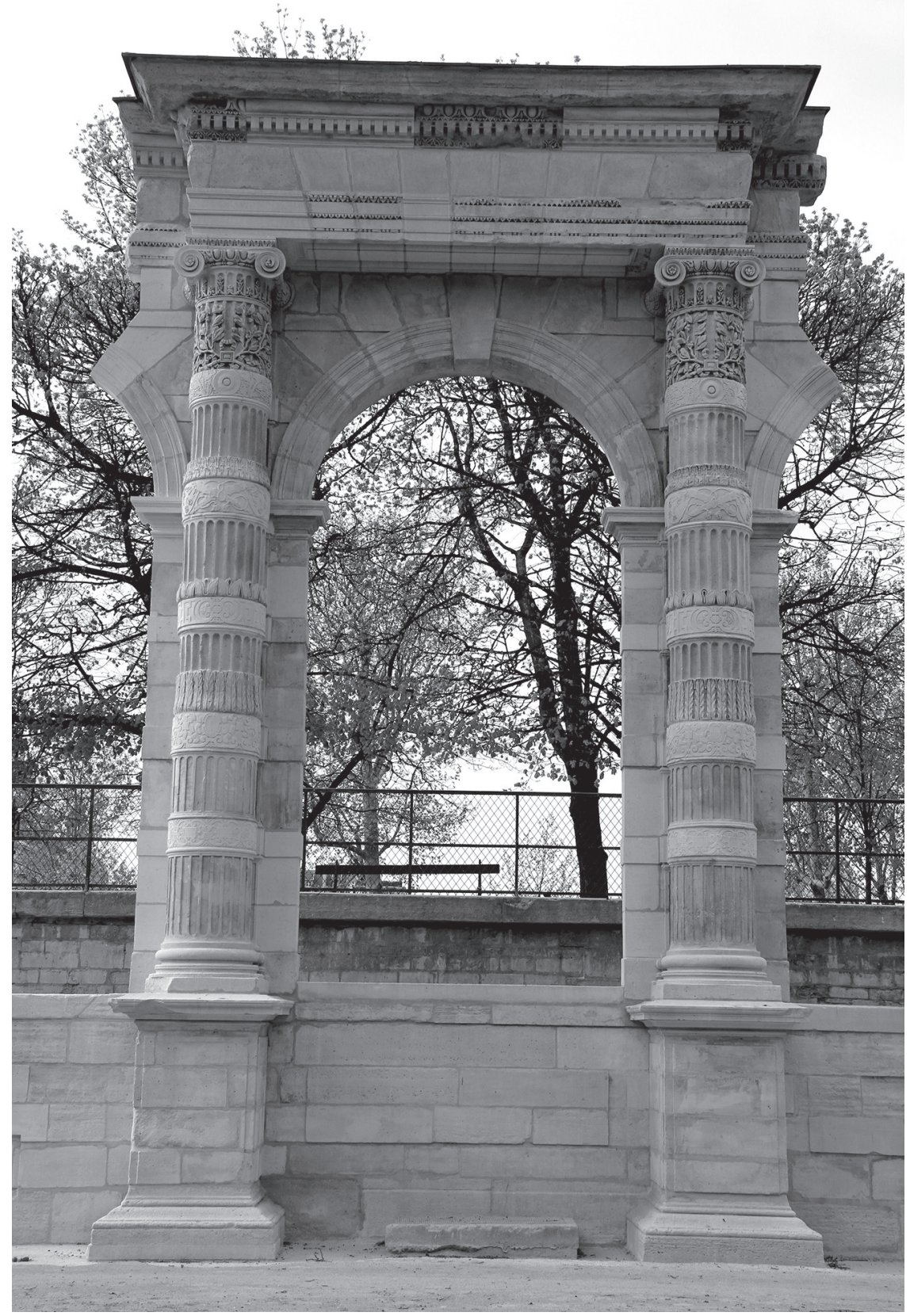

Ill. 1 : Philibert De l'Orme, Paris, arcade des Tuileries remontée dans le jardin (cliché auteur). 
cum capitulo in altitudinem extulerunt. Ita dorica columna virilis corporis proportionem et firmitatem et venustatem in aedificiis praestare coepit ${ }^{3}$.

Quelque temps plus tard, pour édifier un temple à Diane, ces mêmes Ioniens, isdem vestigiis ad muliebrem transtulerunt gracilitatem, et fecerunt primum columnae crassitudinem altitudinis octava parte, ut haberet speciem excelsiorem. basi spiram supposuerunt pro calceo, capitulo volutas uti capillamento concrispatos cincinnos praependentes dextra ac sinistra conlocaverunt et cymatiis et encarpis pro crinibus dispositis frontes ornaverunt truncoque toto strias uti stolarum rugas matronali more demiserunt. ita duobus discriminibus columnarum inventionem, unam virili sine ornatu nuda specie, alteram muliebri subtilitate et ornatu symmetriaque sunt mutuati ${ }^{4}$.

Vitruve raisonne selon un système analogique : les apparences formelles - en l'occurrence le rapport entre le diamètre de la colonne et sa hauteur - évoquent soit les proportions trapues et donc fortes de l'homme, soit celles, plus élancées, de la femme. S'ajoute à cette analogie formelle une distinction ornementale : avec sa base (le dorique n'en est pas pourvu selon le De architectura), les volutes de son chapiteau et les cannelures de son fût, la colonne ionique est ornée, comme l'est l'apparence d'une femme, alors que dans son austérité solide la dorique est plus proche d'une simplicité prétendument masculine: le kouros est nu alors que la korè est vêtue et parée. Quoi qu'il en soit, il sort de ce discours l'idée d'une dualité masculin/ féminin concernant les principaux "genres » de l'architecture, puisque le terme utilisé par Vitruve pour désigner les différentes manières de bâtir un temple est bien genera.

Reste le corinthien, aux proportions proches de l'ionique, qui s'en distingue surtout par son chapiteau inventé selon Vitruve par l'architecte-sculpteur Callimaque. Cette troisième espèce de colonne, dit le théoricien antique,

3. Vitruve, Architecture ou art de bien bâtir, IV, 1, 3. Pour rester dans le contexte de la Renaissance française, je cite la traduction de Jean Martin, Paris, 1547, f. 46 : "ils se prirent à mesurer l'impression de la plante du pied d'un homme; et trouvant que cette mesure faisait une sixième partie de sa hauteur, ils donnèrent cette proportion à leurs colonnes; et de telle largeur qu'était l'étendue du diamètre par en bas, autant de fois voulurent les ouvriers y appliquer cette hauteur, multipliant jusques à six. [...]. Voilà comment la colonne dorique fut premièrement formée sur la proportion du corps de l'homme...".

4. Ibid., f. $47 \mathrm{v}^{\circ}$ : " cherchant une façon nouvelle, ils par semblable invention transportèrent la gaieté féminine à l'usage des colonnes, et tinrent la grosseur de leurs tiges d'une huitième partie de la hauteur, afin qu'elles eussent une espèce plus relevée. En la base ils supposèrent la spire ou bozel en lieu de soulier; et au chapiteau colloquèrent des volutes comme perruques ou chevelures crêpées entortillées et pendantes tant d'un côté que d'autre, puis enrichirent leurs fronts de cimaises ou doucines, les ornant de beaux festons de feuillages pour représenter une tête de femme bien ornée. En outre, tout à l'entour du corps de la colonne depuis le haut jusques au bas, firent des cannelures creuses, afin d'exprimer les plis des vêtements des dames. Et ainsi avec deux inventions différentes parvinrent à l'effet de leur désir, considéré qu'ils en formèrent (comme dit est) une sur la façon du corps mâle (et celle-là nue de tous ornements) puis l'autre sur la délicature de la femme, qu'ils parèrent de beaux ouvrages". 
Tertium vero, quod Corinthium dicitur, virginalis habet gracilitatis imitationem, quod virgines propter aetatis habet teneritatem gracilioribus membris figuratae effectus recipiunt in ornatu venustiores ${ }^{5}$.

Cette idée qui consiste à sexuer les ordres demeure vivante à la Renaissance, période elle aussi déterminée par la pensée analogique ; en outre, elle convient parfaitement aux idéaux de l'humanisme, qui aime à projeter de l'humain dans tous les domaines du savoir, en l'occurrence dans la nouvelle architecture élaborée à Rome au début du $\mathrm{XVI}^{\mathrm{e}}$ siècle, qui renonce aux proportions monstrueuses parce qu'inhumaines de l'art gothique aux piles inconsidérément élancées. La colonne devient une image du corps, avec ses pieds (la base), son corps (le fût) et sa tête (le chapiteau ${ }^{6}$ ), et elle se décline selon les différents genres humains. On retrouve volontiers alors des représentations où un corps est inscrit dans une colonne, ou une tête dans un chapiteau: Francesco di Giorgio en donne plusieurs exemples ${ }^{7}$. Une exception toutefois : Alberti, qui, on le sait, se méfie de Vitruve, préfere dans son De re aedificatoria, rédigé au milieu du $\mathrm{XV}^{\mathrm{e}}$ siècle, renvoyer pour l'origine des proportions des colonnes, à l'Écriture Sainte et aux rapports relatifs au corps de l'homme (que l'on présuppose masculin) tirant leur principe de ceux de l'arche de Noé, pour arriver, à la suite de calculs de moyennes un peu complexes, à des résultats qui sont quasiment les mêmes ${ }^{8}$. Toutefois, cette interprétation n'a que peu d'impact sur la théorie, sinon accidentel ${ }^{9}$. En revanche, la relecture que propose Serlio dans ses Regole generali publiées à Venise en 1537 est d'une autre portée, due à l'efficacité pédagogique de cet ouvrage dont on ne dira jamais assez combien il fut important pour la diffusion du nouveau langage architectural en Europe autour des années 1540-1550.

Serlio reprend donc Vitruve, en le précisant et en apportant des nuances significatives :

Les anciens ont dédié leurs édifices aux dieux, les adaptant à ceux-ci en fonction de leur nature, robuste ou délicate. Ainsi l'œuvre dorique convient-

5. Ibid., f. $48 \mathrm{v}^{\circ}$ : «...fut faite à l'imitation du gent corps de quelque pucelle, pour ce que les filles en leur âge tendre sont de membres grêles et menus, tellement que quand elles sont bien parées, leurs personnes s'en montrent beaucoup plus belles, et d'apparence exquise ».

6. "Capitulum " étant bien évidemment dérivé de "caput ", la tête.

7. Par exemple au folio $13 \mathrm{v}^{\circ}$ du Codex Ashburnham 361 de la Biblioteca Medicea Laurenziana à Florence. L'analogie entre le corps humain et l'architecture en général est récurrente dans l'œuvre de Francesco di Giorgio.

8. Alberti, De re adificatoria, IX, 7. Voir sur ce sujet Yves Pauwels, "Dieu, la nature et les hommes : aux origines de la proportion des ordres dans la théorie architecturale de la Renaissance ", S. Rommeveaux, P. Vendrix et V. Zara (éd.), Proportions. Science, musique, peinture, architecture, Turnhout, Brepols, 2011, p. 395-407.

9. Le texte de la traduction française par Jean Martin (1553) est repris tel quel dans les Reigles generalles d'architecture de Jean Bullant (Paris, Marnef et Cavellat, 1564), mais sans conséquence réelle. Voir Yves Pauwels, "Leon Battista Alberti et les théoriciens français du XVI ${ }^{\mathrm{e}}$ siècle : le traité de Jean Bullant ", Albertiana, II, 1999, p. 101-114. 
elle à Jupiter, à Mars et à Hercule. Ces formes doriques ont été tirées de celles de l'homme; et ils ont utilisé l'ionique pour Diane, Apollon et Bacchus, tirant ses formes de celles des matrones, qui participent à la fois du robuste et du délicat. Car Diane, par sa nature féminine, est tendre, mais l'exercice de la chasse la rend robuste. De même pour Apollon, que sa beauté attendrit, quoiqu'il soit robuste par sa nature masculine; et l'on dira la même chose de Bacchus ${ }^{10}$.

Il ne s'agit plus directement de l'origine des proportions des deux types vitruviens principaux, mais de leur utilisation dans des choix stylistiques correspondant à la nature de leur emploi : le dorique honore les dieux et l'ionique les déesses ou plus largement les divinités "intermédiaires " qui associent les qualités des deux sexes. En effet, Diane est une déesse, mais elle patronne des activités réputées masculines comme la chasse; Apollon et Bacchus sont bien des dieux, mais il y a en eux une part de grâce et de féminité. Serlio intègre dans le système le troisième ordre, le corinthien, qu'il ajoute au dorique viril et à l'ionique "matronal " sous la qualité de "virginal»: "Quant à la manière corinthienne, tirée de la forme des jeunes filles, je voudrais qu'elle soit dédiée à la déesse Vesta, patronne des vierges " ${ }^{11}$. De sorte que la comédie humaine se joue à trois acteurs : le dorique pour les hommes, l'ionique pour les femmes et le corinthien pour les jeunes filles. À partir de là, il devient possible, pour Serlio, d'imiter les Anciens en répartissant les ordres non plus en fonction du genre des dieux, mais du caractère des saints :

Mais, dans cette période contemporaine, il me semble qu'il faut procéder d'une autre manière, sans toutefois dévier de la voie des anciens : je veux dire que, fidèle à nos coutumes chrétiennes, je dédierai, autant que faire se peut, les édifices sacrés à Dieu et à ses saints selon leur apparence ${ }^{12}$.

De sorte que l'on bâtira des édifices doriques pour le Christ et les saints virils et hérö̈ques, saint Pierre, saint Paul, saint Georges, ou " pour d'autres saints comparables, non pas parce que leur profession était d'être soldats, mais qui durant leur vie ont fait preuve de qualités viriles et courageuses les amenant à exposer leur vie

10. Regole generali di architetura sopra le cinque maniere de gli edifici..., Venise, F. Marcolini, 1537, f. 5 : "Gli antichi dedicarono gli edifici à i Dei; accommodandosi à quelli secondo la lor natura robusta, o dilicata: come l'opera Dorica à Gioue, a Marte, \& ad Hercole. Queste si fatte forme Doriche da l'huomo togliendo \& la Ionica à Diana, ad Appolline, \& a Bacco, l'opera togliendo da la forma matronale; che partecipa del robusto, \& del dilicato. Diana, per la natura feminil, e tenera : ma per l'essercitio de la caccia, è robusta : cosi Appolline, per la sua bellezza, è molle : non dimeno è poi robusto, per esser huomo, il medesimo dico di Bacco. "

11. Ibid. : "Ma la maniera Corinthia tolta da la forma verginale, volsero che fosse dedicata alla Dea Vesta presidente à le Vergini".

12. Ibid. : "Ma in questi moderni tempi à me par di proceder per altro modo : non deviando però da gli antichi, voglio dir che seguitando i costumi nostri Christiani, dedicarò, in quanto per me si potrà, gli edifici sacri, secondo le specie loro à Dio, \& a i santi suoi. » 
pour leur foi en le Christ; à tous ceux-ci conviendra ce genre dorique ${ }^{13}$. L'ionique voit sa nature nuancée du fait de l'apparition du troisième ordre corinthien purement féminin : il devient un genre intermédiaire entre les deux extrêmes, qui conviendra "à ces saints dont la vie s'est située entre le robuste et le tendre, et à ces saintes qui auront eu une vie de mère de famille " ${ }^{14}$. Serlio ne dit rien de précis pour le corinthien, mais il est aisé de comprendre qu'il s'adaptera parfaitement aux édifices dédiés aux vierges martyres. Précisons que cette grille de création stylistique genrée, qui est devenue pour nous une grille de lecture, n'est pas unique. La sémantique des ordres intègre de nombreux autre facteurs, d'ordre purement esthétique (du simple au raffiné) ou social (la simplicité dorique convient aux classes les plus modestes, la splendeur du corinthien aux sphères les plus hautes, et le caractère médian du l'ionique à cette classe intermédiaire que sont les lettrés, uomini litterati).

Que devient après Serlio l'idée de l'ordre féminin ? Elle est reprise, nous l'avons vu, par Philibert De l'Orme en 1567, qui y revient à plusieurs reprises ${ }^{15}$. Vincenzo Scamozzi, dans son Idea della architettura universale de 1615, rappelle que l'ionique est "muliebre e di forma grata alla vista " et le corinthien "gracile e virginale " ${ }^{16}$. En Europe du nord, les théoriciens s'en emparent volontiers. Dans son traité des ordres paru pour la première fois en 1550, l'Allemand Hans Blum reprend littéralement le texte vitruvien dans sa dédicace ${ }^{17}$. En 1563, l'Anglais John Shute, dans The first and chief Groundes of Architecture représente sur ses planches à côté des colonnes ionique et corinthienne ordinaires d'autres dont le fût est remplacé par une statue féminine ${ }^{18}$; de même l'Allemand Hans Dietterlin dans son Architectura de $1598^{19}$. Encore plus explicite et franchement moralisateur est un autre Anglais, Sir Henry Wotton, qui en 1624 commente dans ses Elements of Architecture les caractères des ordres, ajoutant au texte antique quelques exigences de décence et étendant son point de vue critique au corinthien, peu recommandable de son point de vue :

13. Regole generali... f. 19 : "ad altri simili santi, che non pur la profession loro sia stata di soldato, ma che habbiano havuto del virile, \& del forte ad esponere la vita per la fede di Christo, a tutti questi tali si convien questa generation Dorica".

14. Regole generali... f. 36v : "a quei santi la vita de i quali sia stata fra'l Robusto \& il tenero. Et cosi a quelle sante, che di vita matronale saranno state ".

15. Cf. ff. 155, 162, 175, 176v, 217.

16. L'idea della architettura universale..., Venise, Vicenzo Scamozzi, 1615, II, lib. 6, chap. 10, p. 32 et 33 .

17. Quinque Columnarum Exacta descriptio atque deliniatio, cum symmetrica earum distributione, Zurich, Christoph Froschauer, 1550, f. 2.

18. The first and chief Groundes of Architecture..., Londres, Thomas Marshe, 1563, f. 7v et 10. Dans le texte, Shute associe le toscan à Atlas, roi "de Mauritanie ", le dorique à Hercule et Mars, l'ionique à Diane ou Apollon, le corinthien à Vesta " or some lyke virgin ", enfin le composite à Pandore (f. Bii). Voir en ligne l'édition de 1563 en fac-similé, https://archive.org/details/firstchiefground00shut (consulté le 8/11/2017).

19. Architectura von Außtheilung, Symmetria und Proportion der Fünff Seulen..., Nuremberg, s.l.s.n., f. 95 . 
L'ordre ionique représente une sorte de sveltesse féminine, à ce que dit Vitruve, mais non pas à la façon d'une ménagère aux mœurs légères, mais décemment habillée, à la manière d'une matrone... La corinthienne est une colonne lascivement parée, telle une courtisane, en quoi elle participe beaucoup (comme le font toutes les inventions) de l'endroit où elle a vu le jour pour la première fois, car Corinthe, sans conteste, fut l'une des villes les plus dévergondées au monde ${ }^{20}$.

Le Grand Siècle classique français, même s'il semble se détacher de ces considérations relevant essentiellement d'une épistémè analogique dont le cartésianisme s'est débarrassé, reste toutefois influencé par les généalogies vitruviennes. Ainsi le très sérieux François Blondel, directeur de l'Académie d'architecture, n'hésite-t-il pas à écrire dans son Cours d'architecture de 1675 que « des modernes ont appelé le Toscan Gigantesque, le Dorique Herculéen, l'Ionique Matronal, le Composé Héroïque, et le Corinthien Virginal ${ }^{21}$.

Qu'en est-il dans la réalité ? Sans doute Vitruve se fait-il l'écho de pratiques réelles en son temps. Certes, on pourrait objecter que le Parthénon d'Athènes, temple d'Athéna, soit d'ordre dorique. Mais la déesse est une guerrière, casquée et armée de sa lance, et ces qualités viriles pourraient expliquer l'emploi de l'ordre masculin - ce qui n'empêche pas le petit temple d'Athéna Nikè d'être ionique. C'est ce que suggère Palladio : "les temples de Minerve de Mars et de Hercule se faisaient d'architecture dorique, estimant que la solidité de cet ordre sans délicatesse convenait aux déités martiales » ${ }^{22}$. Par ailleurs, John Onians analyse le fait que les grands temples grecs soient doriques à l'extérieur mais ioniques ou corinthiens à l'intérieur comme le reflet de la société hellénique, qui gardait les femmes dans l'intimité des demeures en laissant aux hommes l'usage de la place publique ${ }^{23}$. En vérité, il n'existe pas de texte qui corrobore cette interprétation, si séduisante puisse-t-elle paraître. Quant à l'époque moderne, le texte de Philibert est à ma connaissance le seul qui s'applique explicitement à un édifice précis. Il n'en reste pas moins que la sexualisation des ordres permet d'analyser de façon satisfaisante un certain nombre d'emplois de l'ionique. Erik Forsmann, dans son ouvrage fondateur sur l'emploi

20. Henry Wotton, Elements of Architecture, Londres, John Bill, 1624, p. 27-29: "The Ionique Order doth represent a kinde of Feminine slendernesse, yet saith Vitruvius, not like a light Housewife, but in decent dressing, hath much of the Matrone... The Corinthian is a Columne, laciviously decked like a Curtezana, and therein much participating (as all Inventions does) of the place where they first borne : Corinthe having been without controversie one of the wantonest Townes in the world ". Consultable en ligne à l'adresse https://archive.org/details/architectureelem00wott (consulté le $8 / 11 / 2017$ ).

21. Cours d'Architecture, Paris, Lambert Roulland, 1675, première partie, I, III, p. 10. Blondel reprend les qualificatifs de Scamozzi.

22. Il quarto libro dell'architettura, Venise, de'Francesci, 1570, p. 6: " a Minerva, a Marte, \& ad Hercole fecero i Tempii di opera Dorica "; traduction de R. Fréart de Chambray, Les quatre livres d'architecture, Paris, Edme Martin, 1650, p. 196.

23. John Onians, Bearers of Meaning. The Classical Orders in the Antiquity, the Middle Age and the Renaissance, Princeton, Princeton UP, 1988, p. 19-20. 


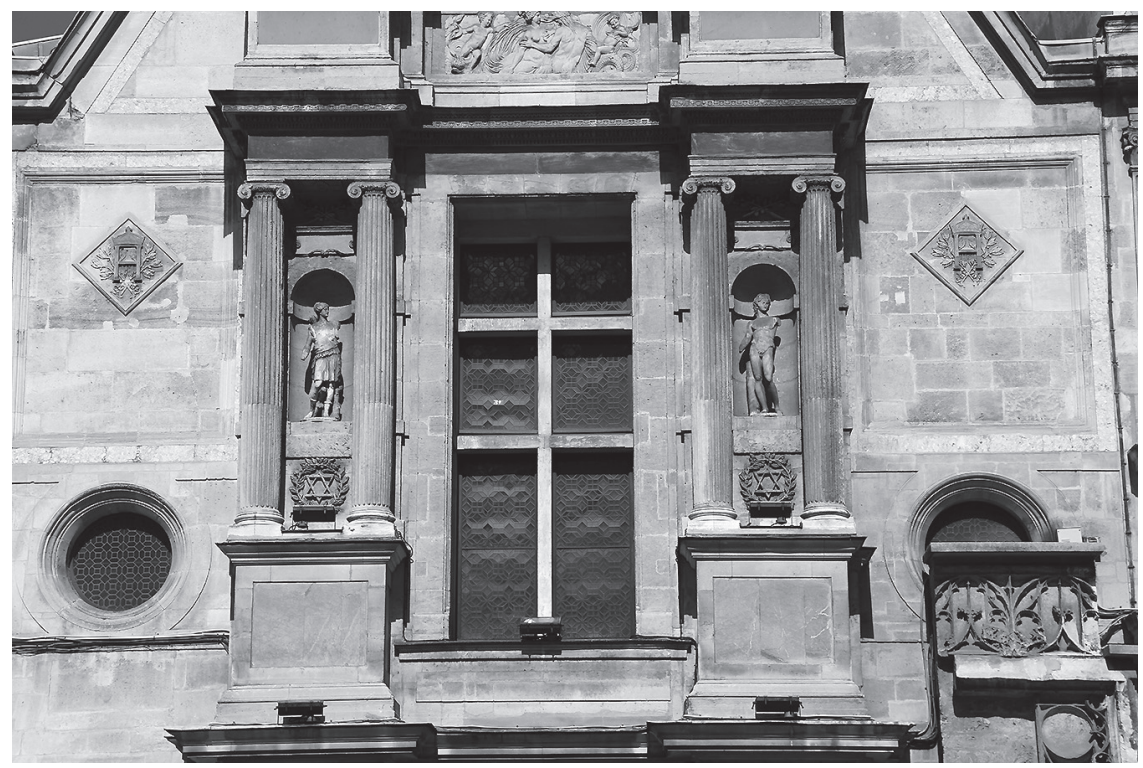

Ill. 2 : Philibert De l'Orme, avant-corps du château d'Anet remonté dans la cour de l'École des BeauxArts à Paris (cliché auteur).

des ordres, analysait l'œuvre de Bramante selon la grille vitruvienne : l'emploi du dorique sur le chœur de la basilique Saint-Pierre ou au Tempietto de San Pietro in Montorio correspond bien au caractère viril et héroïque; l'ionique du cloître de Santa Maria della Pace célèbre dignement la Mère de Dieu, et le corinthien de la Casa Santa de Lorette la jeune femme qui reçoit l'ange dans sa demeure ${ }^{24}$. Pour le reste, les emplois de l'ionique dans l'Italie du Cinquecento, lorsqu'ils ne relèvent pas des cas de superpositions, concernent davantage le caractère "social " de l'œuvre, puisqu'il est l'ordre de prédilection des villas, lieux dédiés au loisir cultivé des uomini litterati $^{25}$.

Le cas de Philibert De l'Orme est plus parlant. Car, si l'on en juge par ce qu'il reste de son œuvre, l'ionique est son ordre favori. Avant de le mettre en œuvre aux Tuileries, il l'a utilisé au second niveau de l'avant-corps de l'aile principale d'Anet, remonté aujourd'hui dans la cour de l'École des Beaux-Arts de Paris, rue Bonaparte. Il s'agit certes ici d'une superposition des trois ordres, où l'ordre féminin est encadré par le dorique et le corinthien, mais dans ce cas, il prend une signification toute particulière car l'étage qu'il orne est celui où résidait Diane de Poitiers (ill. 2). Et

24. Erik Forssman, Dorisch, Jonisch, Korinthisch: Studien über den Gebrauch der Säulenordnungen, Stockholm, Almqvist \& Wiksell, 1961, éd. it., Dorico, ionico, corinzio nell'architettura del Rinascimento, Bari, Laterza, 1973, p. 14-17.

25. À Rome, la villa Madame de Raphaël, la villa Turini Lante de Giulio Romano, la cour de la villa Giulia d'Ammannati et Vasari, la loggia de la villa Médicis, du même Ammannati ; en Vénétie, les villas Foscari à Mira et Capra «Rotonda » à Vicence, toutes deux de Palladio. 


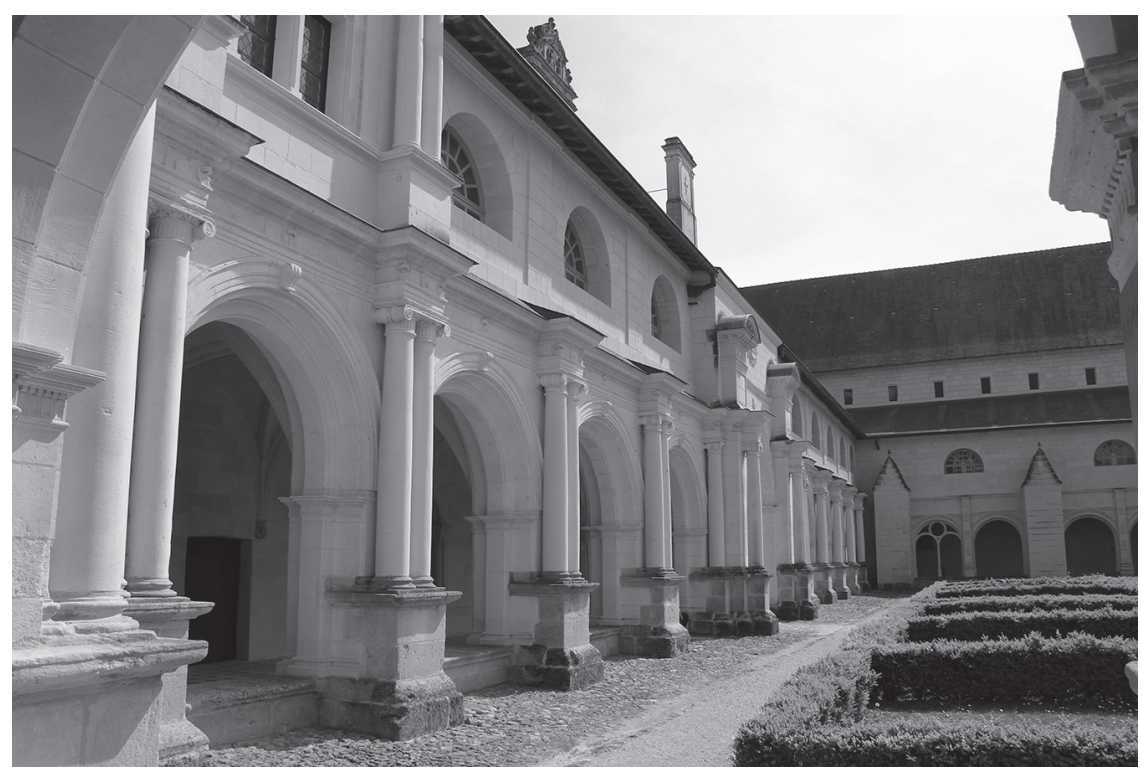

Ill. 3 : Fontevrault, Grand Moûtier, façade extérieure (cliché auteur).

cette coïncidence est d'autant plus pertinente que, comme nous l'avons dit, l'ordre fut inventé à l'occasion de la construction d'un temple de Diane - ce qu'est, dans une version moderne conjuguée au thème du mausolée, le château que du Bellay nomme "Dianet ". La sœur d'Apollon se retrouve en personne sur le relief du châtelet d'entrée : la sculpture, à l'origine une nymphe réalisée par Benvenuto Cellini pour la Porte Dorée de Fontainebleau et recyclée comme déesse de la chasse à Anet, se situe au second niveau de l'édifice, couronnée d'un entablement qu'une frise bombée, dans la gravure du Premier tome, désigne évidemment comme ionique ${ }^{26}$. En même temps, le relief est bordé d'un corps de moulure semi-circulaire interrompu de claveau glyphés que De l'Orme appelle des "triglyphes» - donc des éléments doriques, c'est-à-dire masculin, qui viennent embrasser l'image de Diane, dans une étreinte architecturale qui glose l'inscription placée sous l'œuvre de Cellini ${ }^{27}$.

Moins mythologique est l'emploi de l'ionique dans le Grand Moûtier de l'abbaye de Fontevrault, dont on sait que, réservée aux femmes, elle fut toujours dirigée par une abbesse. Bâti sous la direction de Louise de Bourbon à partir de 1535, le principal cloître est tout naturellement orné sur tout son pourtour d'un ordre ionique (ill. 3). Plus subtilement, cet ionique constitué de colonnes jumelées est conjugué à des pilastres doriques encadrant des niches à l'intérieur des galeries (ill. 4), moins

26. Premier tome..., f. 248 (folioté 247). Cette frise bombée n'existe pas sur le bâtiment dans son état actuel.

27. "Phoebo sacrata est almae domus ampla Dianae verum accepta cui cuncta Diana refert ". Voir $A u x$ marges de la règle, cité note 2 , p. 97-99. 


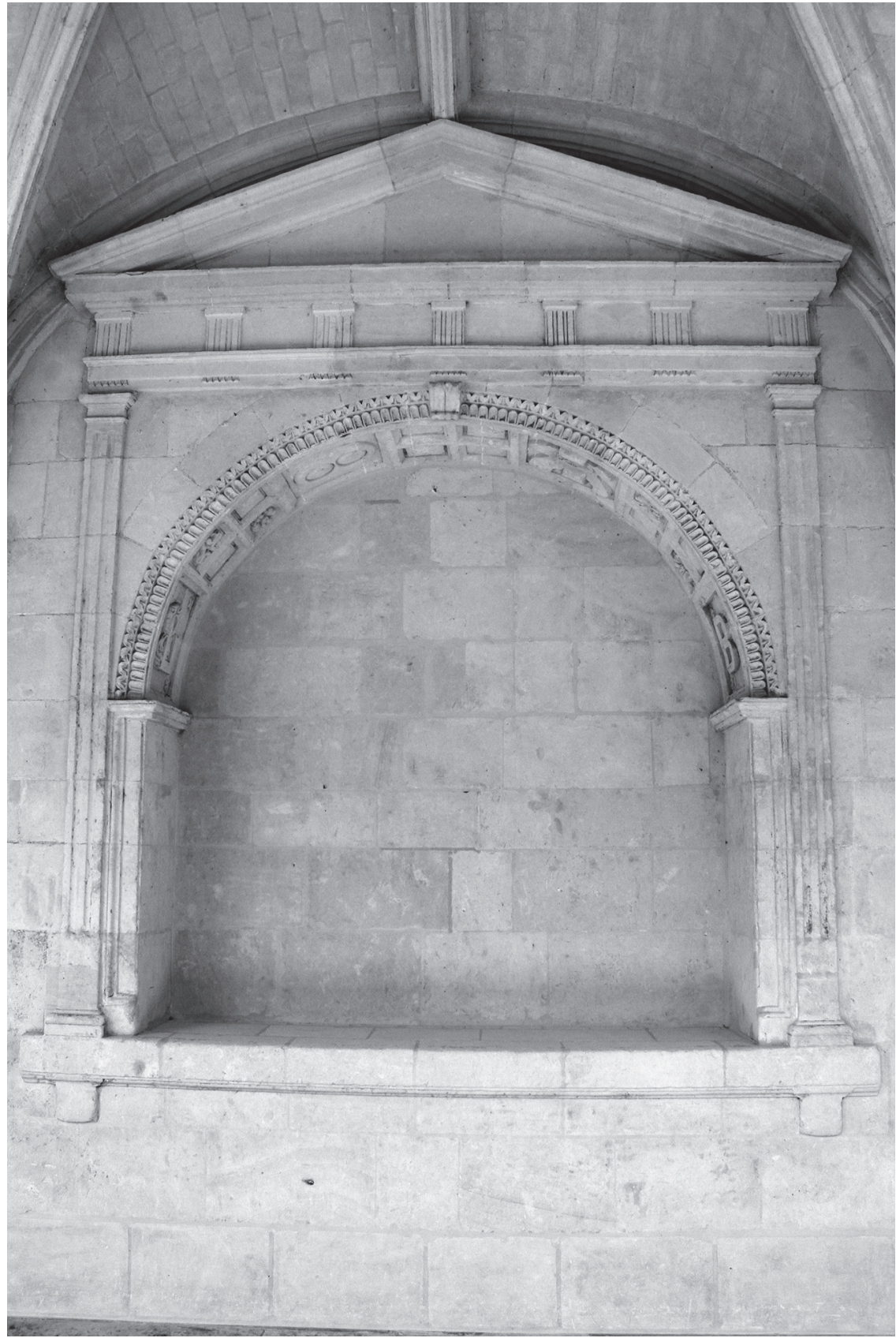

Ill. 4 : Fontevrault, Grand Moûtier, intérieur des galeries du cloître (cliché auteur). 


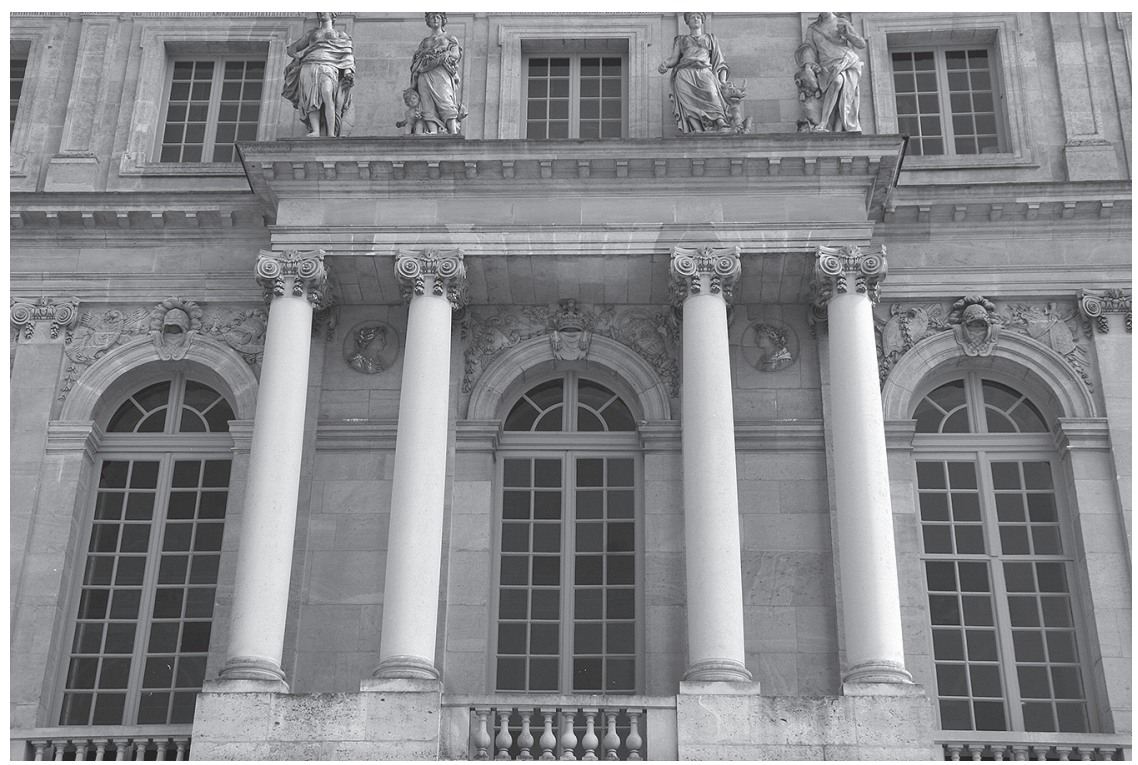

Ill. 5 : Versailles, château, ordre de la façade sur jardin (cliché auteur).

visibles, comme si cette subordination architecturale traduisait la hiérarchie des sexes au sein de l'abbaye royale ${ }^{28}$.

Plus problématique est l'emploi de l'ordre ionique choisi par De l'Orme pour le tombeau de François I ${ }^{\text {er }}$ à Saint-Denis : ce monarque n'a a priori rien de féminin, de sorte que l'ordre ne convient ni d'un point de vue symbolique - il eût fallu un dorique - ni non plus d'un point de vue typologique, car le monument, variation sur le thème de l'arc de triomphe, aurait dû recevoir en tant que tel un corinthien ou un composite. J'ai par ailleurs proposé quelques pistes de lectures qui peuvent rendre ce choix cohérent avec une conception "douce ", quasi féminine, de l'autorité des rois de France, dont les armes s'ornent des lys, fleur mariale, contrairement à celles du roi d'Angleterre ou de l'Empereur, dont les lions ou l'aigle sont beaucoup plus agressifs ${ }^{29}$. Cette douceur des rois de France, qui tempère tout en la sublimant leur autorité, convient à la situation intermédiaire de l'ionique, dont on constate par ailleurs qu'il orne volontiers, au XVII e siècle, les grandes places dédiées à la célébration monarchique, à Paris la place des Victoires et le premier projet de la place des Conquêtes (place Vendôme), ou à Bordeaux la place de la Bourse. Peut-être pourraiton évoquer à ce sujet la façade sur jardin de Versailles, le plus prestigieux des palais royaux, dont l'ordre principal ionique (ill. 5) pourrait surprendre - et de fait, tous

28. Voir Yves Pauwels, "Le mécénat architectural des Bourbon-Montpensier au milieu du XVI e siècle ", Seizième siècle, 12-2016, p. 155-171.

29. Voir Aux marges de la règle..., cité note précédente, p. 160-162, et « Portrait du Roi en architecture : le tombeau de François I ${ }^{\text {er }}$ à Saint-Denis ", B. Petey-Girard, G. Polizzi et T. Tran (éd.), François I ${ }^{e r}$ imaginé, Genève, Droz, 2017, p. 395-408. 
les grands châteaux qui s'en inspirent, de Caserte à Saint-Pétersbourg ${ }^{30}$, succombent logiquement aux fastes du corinthien ou du composite. Mais la demeure est aussi un temple d'Apollon, qui règne sur le grand axe du jardin, en compagnie de Diane dans le bassin de Latone. Rappelons-nous que Serlio dédiait «la Ionica à Diana, ad Appolline, \& a Bacco, l'opera togliendo da la forma matronale "... ${ }^{31}$ L'ionique de Versailles est un ordre apollinien, qui a sa part de féminin.

Yves Pauwels

professeur d'Histoire de l'art moderne

Tours, centre d'études supérieures de la Renaissance université François Rabelais

30. Seul Schönbrunn présente un ordre ionique dominant (interrompu par un composite sur l'avantcorps central côté cour). Mais il s'agit du palais de Marie-Thérèse.

31. Voir plus haut, note 7. 